\title{
Video-imaging of lumen narrowing; muscle shortening and flow responsiveness in isolated bronchial segments of the pig
}

\author{
H.W. Mitchell, M.P. Sparrow
}

Video-imaging of lumen narrowing; muscle shortening and flow responsiveness in isolated bronchial segments of the pig. H.W. Mitchell, M.P. Sparrow. CERS Journals Ltd 1994. ABSTRACT: The amount of smooth muscle shortening required to cause narrowing of the bronchial lumen and to significantly alter airflow in an airway has not been measured directly.

Accordingly, in this study, the lumens of isolated fluid-filled bronchial segments from pigs were directly visualized using a fibreoptic endoscope and video-recording. The extent of lumen narrowing was related to smooth muscle contraction, determined morphometrically, and to bronchial lumen flow. Narrowing was produced by electrical field stimulation (EFS), $(70 \mathrm{~V}, 20 \mathrm{~Hz}, 2-3 \mathrm{~ms})$ and acetylcholine (ACh), $\left(10^{-5}-10^{-2} \mathrm{M}\right)$.

The diameter of the relaxed bronchial lumen and the reduction in diameter produced by maximum electrical field stimulation were both greater when the transmural pressure was increased (from -8 to $20 \mathrm{cmH}_{2} \mathrm{O}$ ). The percentage change in diameter produced by electrical field stimulation was not, however, significantly different over this pressure range. Electrical field stimulation caused the bronchial lumen to narrow by $28.5 \pm 3.3 \%$ in diameter, and by $45.7 \pm 3.8 \%$ in cross-sectional area, at zero transmural pressure. Maximum doses of acetylcholine $\left(10^{-2} \mathrm{M}\right)$ reduced the lumen diameter by $48.3 \pm 3.3 \%$, and the area by $70.3 \pm 4.8 \%$. In separate experiments, maximum doses of ACh stopped the flow of Krebs solution perfused through the lumen of bronchial segments. Morphometric measurements indicated that smooth muscle shortened by a maximum of $32.5 \pm 2.8 \%$ to acetylcholine $10^{-2} \mathrm{M}$, relative to fully-relaxed dimensions. Simultaneous imaging of the lumen and adventitia of bronchi showed that the luminal margin narrowed more than the adventitia.

The use of direct video-imaging has shown that functional airway closure is associated with an approximate halving of the lumen diameter but less shortening of the smooth muscle. The greater movement of the luminal than adventitial margins suggests that the smooth muscle and mucosa move independently of the cartilaginous part of the bronchial wall during airway narrowing.

Eur Respir J., 1994, 7, 1317-1325.
Dept of Physiology, University of Western Australia, Nedlands, Australia.

Correspondence: H.W. Mitchell Dept of Physiology

University of Western Australia

Nedlands 6009

Australia

Keywords: Airway smooth muscle bronchi

bronchial responsiveness

video-imaging

Received: July 301993

Accepted after revision March 301994

The work was supported by the National Health and Medical Research Council of Australia.
The extent of airway narrowing throughout the bronchial tree during bronchial provocation is usually inferred from measurements of flow or total airway resistance made at the mouth. Direct estimates of narrowing at particular sites in the lung in vivo or in situ have been made from images of the bronchial lumen obtained by radiographs using tantalum [1-5], or recently by high-resolution computed tomography [6,7]. These suggest that the maximum airway narrowing to drug or nerve stimulation of the airway smooth muscle ranges from $20-50 \%$ in lumen diameter, or $75 \%$ or more in lumen crosssectional area, at different transpulmonary pressures and airway locations $[2-4,6]$. The change in flow in an individual airway produced by this degree of narrowing, however, has not been established, since flow, measured at the mouth, results from the combined actions of airways in series and parallel throughout the lung.
Although changes in the bronchial lumen are produced by airways smooth muscle contraction, there are no direct measurements defining the relationship between bronchial flow and smooth muscle length in airways. Studies in the dog trachea suggest that maximum smooth muscle shortening in situ is only about 20-30\% [8]. However, the narrowing produced by the contraction of the smooth muscle is probably influenced by the airway wall, which may occlude the lumen progressively [9-11] as the stimulus to the smooth muscle increases. The folding of the mucosa may also influence narrowing by stabilizing the bronchial wall [12], or it may limit the area available for flow if luminal fluid becomes trapped in the interstices produced during narrowing [13]. Although the above properties of the airway wall may contribute to the overall reduction in lumen area produced by smooth muscle contraction in all airways, their impact may vary with 
airway size $[10,11,14]$, or with pathological changes in airway morphology $[9,10]$.

In isolated perfused bronchial segments from animals $[14,15]$ and man $[16,17]$, maximal cholinergic stimulation reduces luminal flow by $80-100 \%$. We reasoned that the bronchial segment might provide an experimental model of airway responsiveness, which would enable us to determine the extent of lumen reduction brought about by submaximal and maximal smooth muscle shortening. The objective was to define the relationship between airway narrowing, measured directly, and smooth muscle shortening. We therefore applied fibreoptic endoscopy and video-imaging techniques to visualize the luminal and adventitial surfaces of bronchial segments, in order to determine narrowing in response to cholinergic stimulation. Measurements were made at zero transmural pressure, and also after raising or lowering transmural pressure in order to simulate airway conditions in vivo.

\section{Methods}

Physiological experiments were carried out on airways from 16 pigs, obtained immediately after slaughter. Pigs were killed with a captive bolt gun and exsanguinated.

\section{Preparation of bronchial segments}

The lungs were removed and the bronchi were dissected out on ice as described previously [15]. After ligating all side-branches, we cannulated a $2-3 \mathrm{~cm}$ length of the "stem" bronchus with an internal diameter of about 3 $\mathrm{mm}$. The segment was placed horizontally in an organ chamber. Each end of the segment was securely fixed to a Perspex inlet or outlet built into the organ chamber, so that the length of the segment could not change (fig. 1). The adventita (i.e. outside) of the bronchial segment was bathed in gassed Krebs solution at $37^{\circ} \mathrm{C}$, whilst the lumen (i.e. inside) could be flushed through with Krebs solution. The solutions were refreshed regularly and drugs (acetylcholine (ACh)) could be added to the solution bathing the outside of the segment.

\section{Visualization of lumen narrowing}

To visualize the lumen of the bronchial segment, we used a rigid fibreoptic endoscope (Olympus Selfscope SES 1711D). The diameter of the endoscope was 1.7 $\mathrm{mm}$, and the lens had a $60^{\circ}$ forward field of view. The endoscope image was recorded with an Ikegami colour CCD video-camera (type ICD 840P). The output from the camera was recorded on video-tape for subsequent image analysis (see below).

Camera and endoscope were mounted on a moveable platform, which allowed the tip of the endoscope to be gently inserted into the segment lumen via the organ chamber outlet (fig. 1). To aid the visualization of a particular region of the lumen circumference, we first applied a small amount of dye (amidoschwarz, Merck Laboratories) to the bronchial mucosa. This was done using an applicator made from a stainless steel tube with an enlarged spherical head $3 \mathrm{~mm}$ in diameter, containing perforations through which the dye was gently forced under a small positive pressure. This dye bound well to the mucosa for several hours, during which time the experiment was run. If the ring of dye was found to be grossly irregular in shape, then another area of dye was applied to obtain a suitable recording.

The diameter and area of the lumen was measured by playing back the video-tape through a chromatic image analysis system (Leading Edge, Pty Ltd, Bellview Heights, South Australia). The image was calibrated with a 1 $\mathrm{mm}$ diameter probe, which was inserted into the distal end of the segment and advanced until it was exactly adjacent to the region of dye on the bronchial mucosa. The functional luminal area was measured from a circle drawn over the inner (luminal) margins of the mucosa but excluding the interstices. The excluded area constituted less than $6 \%$ of the total area, including interstices. The effective diameter of the region of interest was calculated from the perimeter of the traced area, assuming that it was circular (see below). The intraobserver variability was determined from duplicate measurements of the perimeters of five different airways, and it was $0.65 \pm 0.62 \%(n=5)$.

To test how close the shape of the region of interest was to a circle, in each relaxed and contracted bronchus,

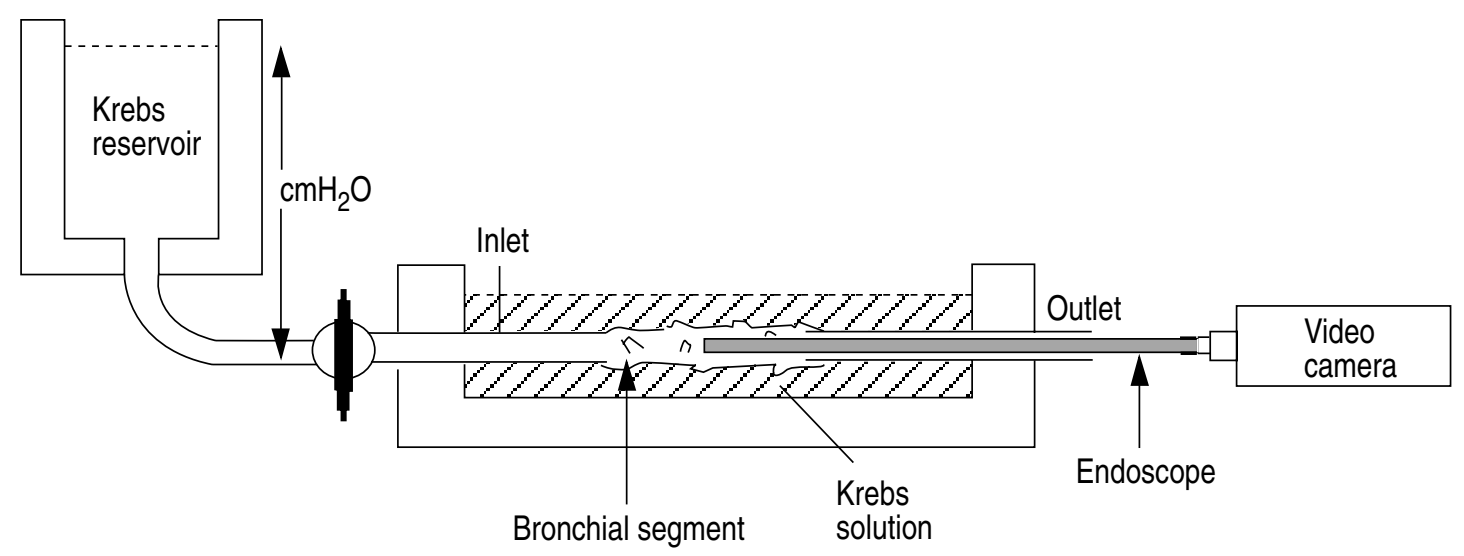

Fig. 1. - Diagram indicating arrangement of bronchial segment in the organ chamber, the endoscope inserted into the bronchial lumen, and Krebs solution reservoir with tap by means of which the transmural pressure $\left(\mathrm{cmH}_{2} \mathrm{O}\right)$ could be varied; see text for explanation. Platinum ring electrodes were built into the organ chamber (not shown) for delivering electrical stimulation. 
we compared the lumen cross-sectional area actually measured with the endoscope system with the area of a true circle calculated from the perimeter traced from the video image of that airway. The results (fig. 2) show a strong correlation between calculated and measured luminal areas, with a slope very close to unity. Results were the same for both relaxed and contracted bronchi, indicating that the traced lumen was circular in either state.

Narrowing responses were obtained by electrical field stimulation (EFS) of the bronchus via platinum ring electrodes, using a Grass S44 square wave stimulator (EFS; $70 \mathrm{v}, 20 \mathrm{~Hz}, 2-3 \mathrm{~ms}$ ), or by adding ACh to the Krebs solution bathing the outside of the bronchial segment.

Experimental measurements were made under isobaric conditions, after closing a tap between the Krebs solution reservoir and the organ chamber inlet (fig. 1) so that

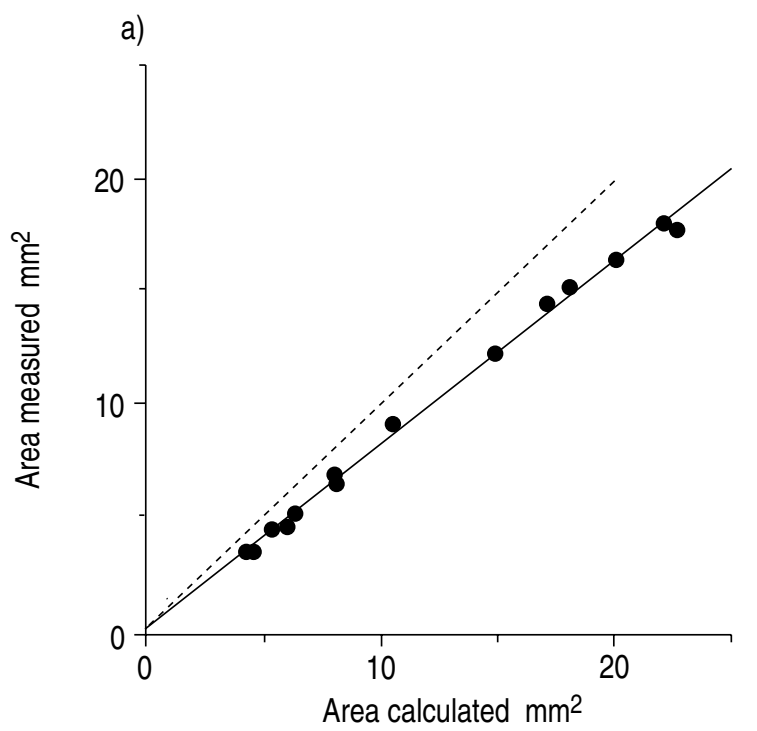

b)

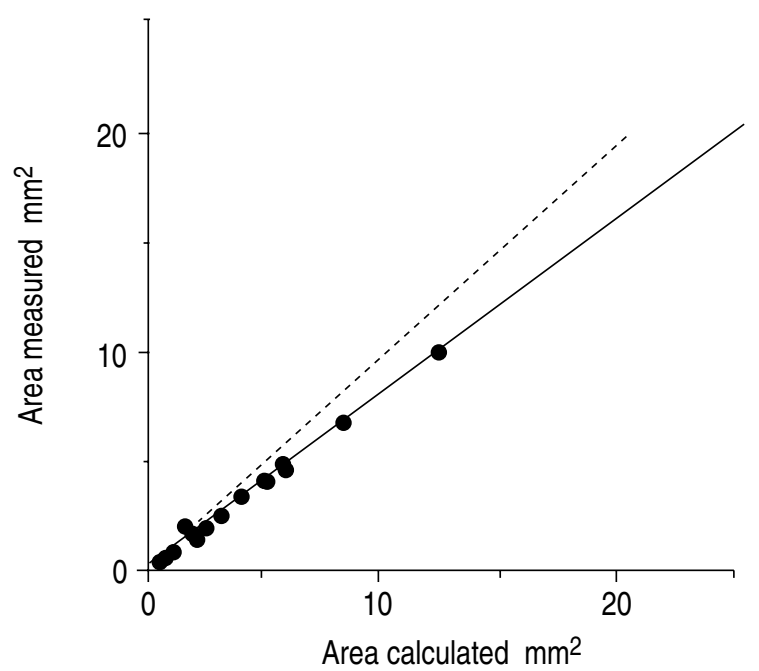

Fig. 2. - Comparison of lumen cross-sectional area measured directly from the endoscopic image with the area calculated from the perimeter (perimeter $2 / 4 \pi)$ of the lumen of different bronchial segments $(n=16)$. a) data for relaxed bronchi $(\mathrm{r}=0.9984 ; \mathrm{p}<0.001)$. b) data during constriction to acetylcholine $\left(10^{-5}-10^{-2} \mathrm{M}\right)(\mathrm{r}=0.9996 ; \mathrm{p}<0.001)$. The predicted relationship (dashed line) for a circle is shown. there was no flow through the bronchial lumen. Most experiments were performed when the transmural pressure was $0 \mathrm{cmH}_{2} \mathrm{O}$, i.e. with the organ chamber outlet, through which the endoscope was inserted, open to the atmosphere. In some runs, however, we studied the effect of varying transmural pressure on lumen narrowing to EFS. This was done by raising or lowering the reservoir containing Krebs solution, with the reservoir tap open but with the outlet end of the segment temporarily sealedoff with Plasticine to maintain the lumen pressure. In this manner, EFS responses were obtained at $-8,0,10$ and $20 \mathrm{cmH}_{2} \mathrm{O}$ transmural pressure. In these experiments, diameters of both lumen and adventitia of the bronchi were recorded on video-tape. Adventitial margins were visualized via a dissecting microscope placed above the bronchial segment.

\section{Bronchial flow}

In separate experiments on four bronchial segments, the flow of Krebs solution through the lumen in response to EFS or ACh was recorded using a system based on that described previously [15]. Segments were mounted in apparatus which incorporated an organ chamber similar to that used in the endoscopy experiments. The lumen was perfused at a constant driving pressure with Krebs solution from a reservoir set $5 \mathrm{~cm}$ above the bronchus. Flow through the lumen was measured using a rotameter combined with a Hall Effect detection device (Airway Narrowing Analyser, Ugo Basile Biological Research Apparatus, Comerio-Varese, Italy). The downstream resistance of the rotameter was less than $30 \%$ of the resting bronchial resistance. After equilibrium, bronchi were stimulated several times with EFS, with 5 min intervals between trains of impulses, and then upon recovery by supramaximal ACh $10^{-2} \mathrm{M}$.

\section{Morphometry}

Bronchial segments used to visualize the lumen were fixed in the organ chamber by addition of formaldehyde (at a bath concentration of $4 \%$ ), to the Krebs solution bathing the segment. This procedure had no effect on the lumen diameter as detected with the endoscope. After at least $20 \mathrm{~min}$, the tissue was carefully removed from the chamber and returned to buffered fixative for $24 \mathrm{~h}$, after which time it was placed in Cryo-M-Bed and frozen in isopentane cooled in liquid nitrogen. Cryopreparation was used in order to avoid tissue shrinkage caused by wax embedding. Multiple serial cryosections $(15 \mu \mathrm{m})$ were cut along $0.3 \mathrm{~mm}$ of the segment at the region of interest, and these sections were then stained with haematoxylin and eosin.

Sections were examined microscopically using the chromatic image analysis system [14]. The cross-sectional area of the inner wall (mucosa and smooth muscle) was determined by subtracting the area of the lumen from the combined area of the lumen and the airway wall out to the adventitial margin of the smooth muscle. The 
relative wall area was the area of the inner wall, expressed as a fraction of the total area of the airway cross-section to the adventitial margin of the smooth muscle, assuming circularity of the epithelium in the relaxed state [18].

Smooth muscle shortening was estimated morphometrically using the procedure described by JAMES et al. [18]. The smooth muscle extended for more than $90 \%$ of the airway circumference. Active smooth muscle shortening to ACh was corrected by subtracting the value of shortening obtained in relaxed unstimulated bronchi, also at $0 \mathrm{cmH}_{2} \mathrm{O}$ transmural pressure, i.e. the same pressure used for the ACh runs. This was $8.8 \pm 1.4 \%(n=6)$.

\section{Protocol and treatment of data}

Bronchial segments were left to equilibrate in the organ chamber for $1 \mathrm{~h}$. After applying the dye to the mucosa, the endoscope was inserted and the segment underwent EFS several times with 5 min intervals between trains. The stimulus parameters $(70 \mathrm{~V}, 20 \mathrm{~Hz}, 2-3 \mathrm{~ms}$, for $15-$ $30 \mathrm{~s})$ gave maximum response. In some experiments, the transmural pressure was then varied, as outlined above, and EFS responses were recorded at each pressure. Lastly, segments were stimulated with $\mathrm{ACh}$ and the narrowing response of the airway was monitored until after it reached steady-state (usually $>5 \mathrm{~min}$ ). At steady-state the segment was fixed for histological processing as described.

Lumen narrowing was measured from the signals recorded on video-tape (see above). Luminal cross-sectional area and diameter were measured, using the calibrated system, immediately before EFS or ACh and again at the peak of the response. For EFS, responses of duplicate or triplicate runs in each segment were averaged. Single responses to ACh were recorded in each segment. Dimensions ( $\mathrm{mm}$ diameter or $\mathrm{mm}^{2}$ area) of the segments relaxed and contracted were compared by a paired t-test.

A predicted relationship between muscle shortening and luminal area or diameter of live bronchial segments was determined using Microsoft Excel. This was calculated from the fractional circumferential shortening of the smooth muscle and from a relative area of the inner wall (mucosa and smooth muscle) of 0.16 , which was determined from morphological measurement of fixed tissue (see above and table 1). Correlation coefficients were calculated by the method of least squares. A relationship between different transmural pressures versus lumen diameter and between inside versus outside diameters during EFS was analysed by two-way analysis of variance (ANOVA). In addition, a predicted reduction of the luminal diameter of the bronchus during stimulation with EFS was calculated from the recorded reduction in diameter of the outer adventitial surface of the bronchus and from the total bronchial wall area (mucosa, smooth muscle and cartilage). The total bronchial wall area was determined from the difference between the total bronchial cross-sectional area determined from the adventitial diameter, and the crosssectional area of the lumen determined from the lumen diameter. Data shown are mean \pm SEM, with $n=$ number of bronchial segments. A p-value of $<0.05$ was considered significant.

\section{Results}

With adequate lighting, the features of the airway lumen were readily visualized, with lines between the mucosal ridges or folds, and side-branches, clearly identifiable. The number of mucosal folds in bronchial segments from different pigs was highly consistent and averaged $23 \pm 1$ $(n=6)$. This was the same $(24 \pm 1)$ as the number of folds observed in fixed tissue used in the morphometric estimation of muscle shortening (see below). Examples of images from different bronchial segments are shown in figures 3 and 4.

Occasionally, side-branches were close to the region of interest, but these did not appear to cause a significant distortion in the narrowing response; for example, in figure 3 , narrowing was essentially concentric despite the presence of a large side-branch. It is clear from figure 3 that the opening to the side branch also narrows during EFS.

The following results were obtained from regions of the bronchus that were distal to side-branches, so that the line drawn across the margins of the mucosal ridges was almost circular (fig. 2). Unless noted otherwise, measurements were made at $0 \mathrm{cmH}_{2} \mathrm{O}$ transmural pressure.

\section{Narrowing to EFS and acetylcholine}

EFS $(70 \mathrm{~V}, 20 \mathrm{~Hz}, 2-3 \mathrm{~ms})$ caused the lumen to narrow. The onset of the narrowing response was almost immediate $(<2 \mathrm{~s})$, and maximum narrowing occurred at 15-30 s. At the end of EFS, the lumen dilated rapidly and completely in about $15 \mathrm{~s}$ (fig. 3).

Table 1. - Dimensions of relaxed and contracted bronchial segments

\begin{tabular}{|c|c|c|c|c|c|c|}
\hline & \multicolumn{2}{|c|}{ Area $\mathrm{mm}^{2}$} & \multicolumn{2}{|c|}{ Diameter $\mathrm{mm}$} & \multirow{2}{*}{ WA mm² } & \multirow[t]{2}{*}{ rWA } \\
\hline & Morphometry & Endoscopy & Morphometry & Endoscopy & & \\
\hline Relaxed & $8.02 \pm 0.64$ & $10.02 \pm 1.60$ & $3.78 \pm 0.07$ & $3.63 \pm 0.33$ & $2.00 \pm 0.19$ & $0.18 \pm 0.02$ \\
\hline Contracted & $2.91 \pm 0.79 * *$ & $3.30 \pm 0.86^{*}$ & ND & $2.00 \pm 0.27 * *$ & $2.14 \pm 0.07$ & $0.16 \pm 0.01$ \\
\hline
\end{tabular}

Dimensions of bronchial segments determined firstly by video-imaging (cross-sectional area and diameter of the lumen) and after histological processing for morphometry of the same airway preparation. WA: cross-sectional area of bronchial wall from the epithelium to the adventitial margin of the smooth muscle determined by morphometry; rWA: relative wall area (WA relative to the relaxed WA and lumen area combined). Segments were studied either without treatment (relaxed, $\mathrm{n}=4)$ or maximally contracted with acetylcholine $(\mathrm{n}=13)$. All measurements were made at zero transmural pressure. $*: \mathrm{p}<0.01 ; * *: \mathrm{p}<0.001$ compared with relaxed. ND: not determined. 

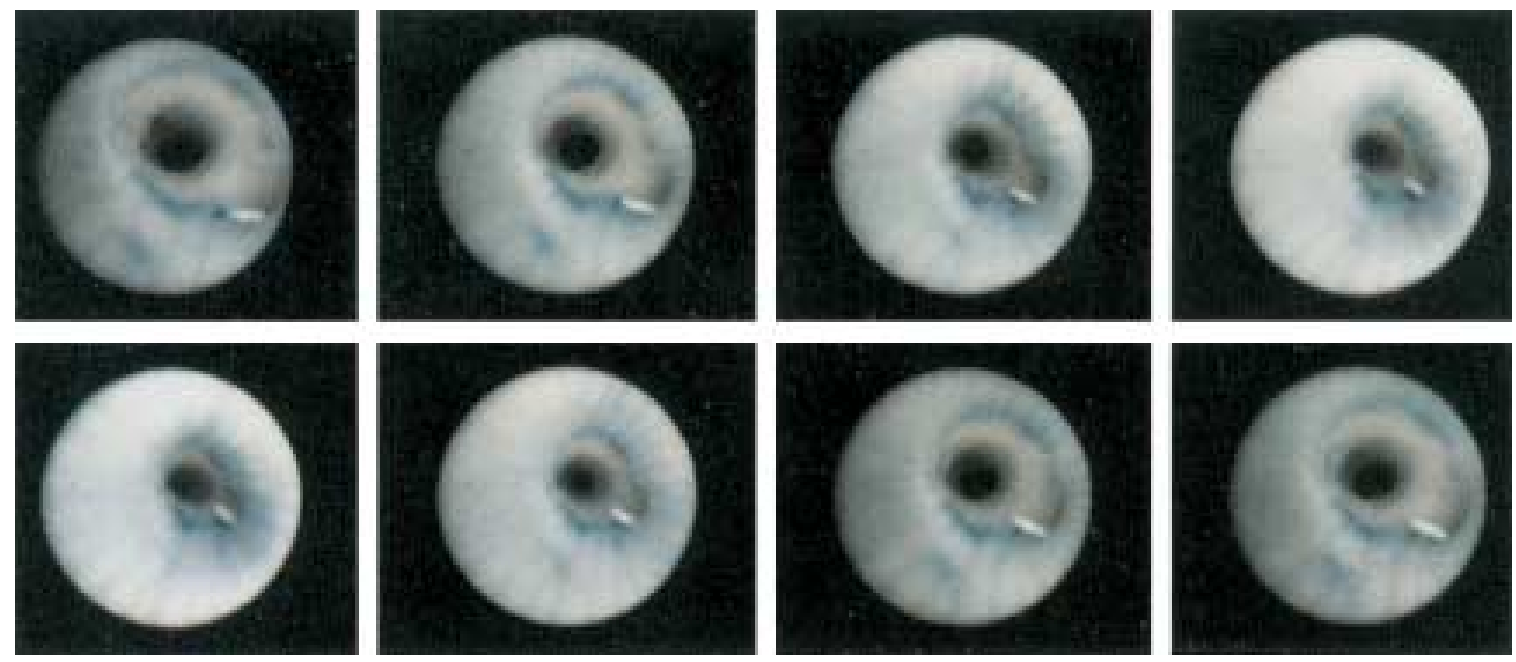

Fig. 3. - Video images along a bronchial lumen during narrowing in response to electrical field stimulation (EFS) (70 V, $20 \mathrm{~Hz}, 2 \mathrm{~ms})$. A circle of blue dye was applied around the lumen in the vicinity of a side-branch and the diameter in this region was $3 \mathrm{~mm}$. Both the lumen and adventitia of the bronchial segment were illuminated, and a region of bright reflection is visible at the entrance of a side-branch. The sequence shows, from left to right, video frames at $6 \mathrm{~s}$ intervals during EFS. Top left frame was taken immediately before stimulation. The stimulator was turned-off at the fifth frame of the sequence (i.e. at $24 \mathrm{~s}$ ). Note the rapidity of the narrowing and relaxation phases of the response, the mucosal foldings which do not change in number during narrowing, and the narrowing of the entrance to the side-branch during stimulation.
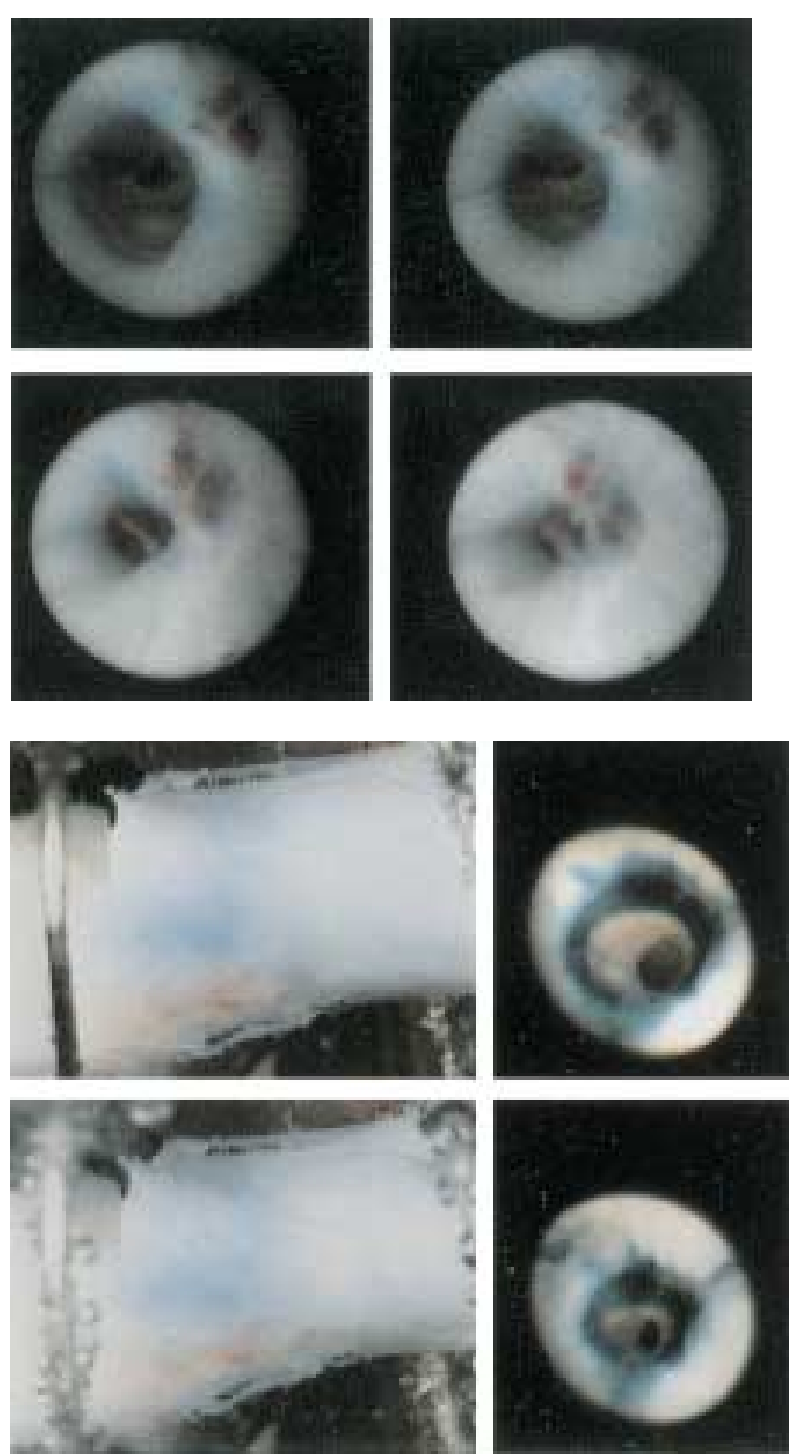

Fig. 4. - Narrowing to acetylcholine, $10^{-2} \mathrm{M}$, in a $2.7 \mathrm{~mm}$ internal diameter bronchial segment. Top left frame shows airway before drug stimulation and subsequent frames (left to right) are at 1 min intervals. Maximum narrowing was present after 3 min. A small piece of mucus moved into the distal lumen during the narrowing response.

Fig. 8. - Video imaging of the adventitia of a pig bronchial segment and of the lumen of the same segment recorded via an endoscope. The upper panels show the unstimulated airway and the lower panels during electrical field stimulation (EFS). Adventital control diameter $=5 \mathrm{~mm}$, and lumen $=3.3 \mathrm{~mm}$. EFS caused the lumen to narrow by approximately $40 \%$, whilst the adventita narrowed by about $10 \%$. 
With $\mathrm{ACh}$, in contrast, narrowing was slow (2-5 min). In a few experiments, the lumen became slightly oval in shape during maximum narrowing. In the majority of runs, however, the constricted lumen maintained its circular appearance (fig. 4).

The cross-sectional area and diameters of the bronchial lumens relaxed and contracted during $10^{-2} \mathrm{M} \mathrm{ACh}$, are shown in table 1 . This maximum dose of $\mathrm{ACh}$ reduced the area of the lumen by $70.3 \pm 4.8 \%$, and the diameter by $48.3 \pm 3.3 \%$. EFS produced less of a response, $45.7 \pm 3.8 \%$ $(\mathrm{n}=18 ; \mathrm{p}<0.001)$ reduction in area, and $28.5 \pm 3.3 \%(\mathrm{n}=18$; $\mathrm{p}<0.001)$ in diameter.

In separate experiments, we determined the effect of EFS and ACh on the flow of Krebs solution through the lumen of perfused bronchi (fig. 5). EFS caused a $68 \pm 6 \%$ reduction in flow $(n=4 ; p<0.001)$. On the other hand, high concentrations of ACh $\left(10^{-2} \mathrm{M}\right)$ always caused flow to stop in pig bronchial segments.

\section{Shortening of smooth muscle and lumen narrowing}

At the height of an ACh contraction, bronchi were fixed for histological processing and estimation of in situ smooth muscle shortening. Morphometrically determined areas and diameters of the lumen and the wall area and relative wall area are presented in table 1 . The dimensions of the lumen of relaxed and contracted bronchi measured morphologically were the same as those determined endoscopically.

Maximum muscle shortening to $10^{-2} \mathrm{M}$ ACh was $32.5 \pm 2.8 \%(n=9)$. In further experiments, we measured airway narrowing and muscle shortening after stimulation with submaximal concentrations of ACh (from $10^{-5} \mathrm{M}$ ). The experimentally obtained values for maximal and submaximal stimulation are compared with a predicted curve, which takes into account the effect of the inner airway wall (see methods) on lumen narrowing (fig. 6).

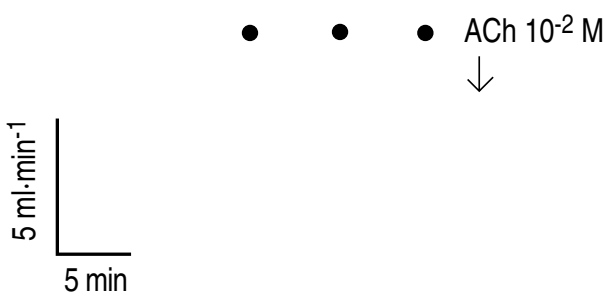

Pig

\section{Zero flow}

Fig. 5. - Effect of electrical field stimulation (EFS) and acetylcholine (ACh) on flow through the lumen of a perfused bronchial segment under a driving pressure of $5 \mathrm{cmH}_{2} \mathrm{O}$. EFS $(70 \mathrm{~V}, 20 \mathrm{~Hz}, 2 \mathrm{~ms})$ was applied where indicated $(\bullet)$ for approximately $30 \mathrm{~s}$, which produced the maximum response. After several responses to EFS, ACh was added to the organ chamber. ACh always produced cessation of flow.
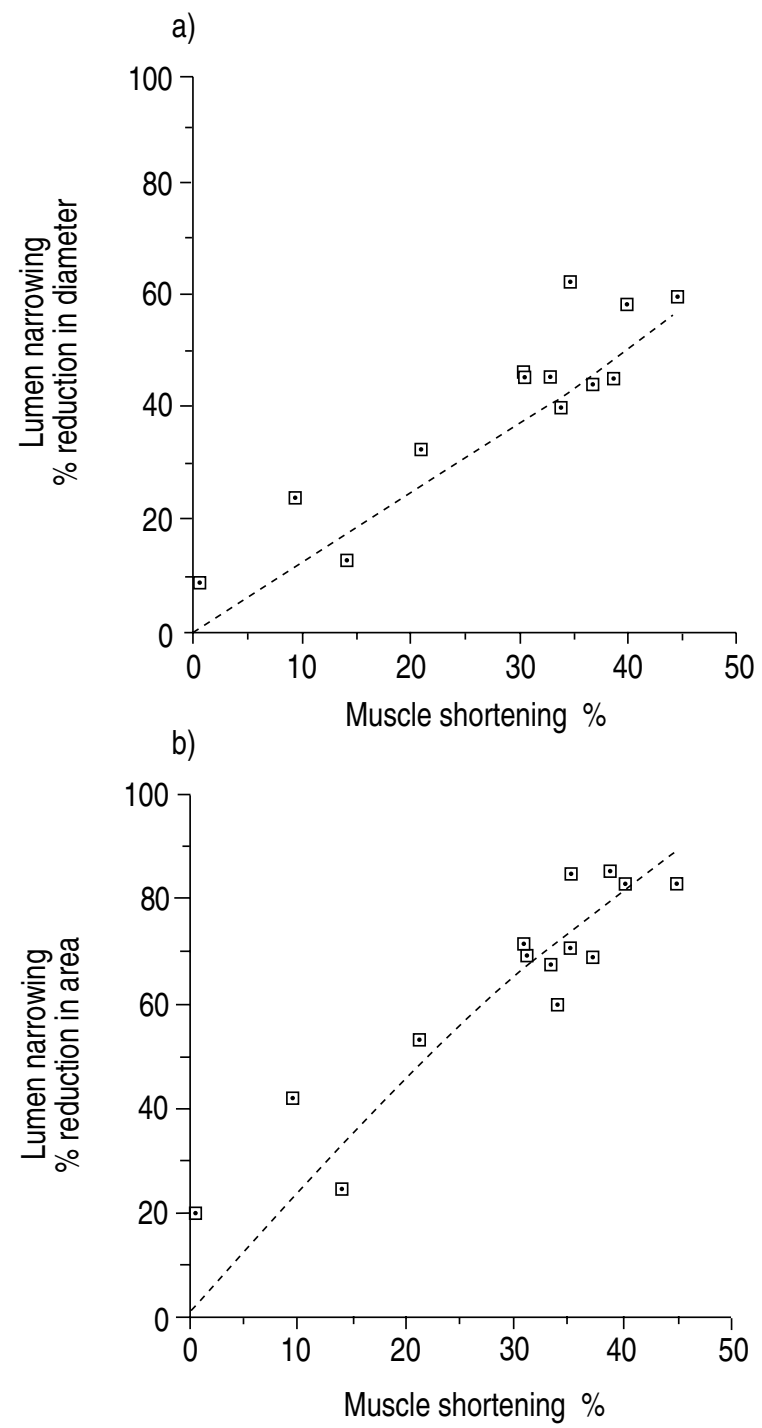

Fig. 6. - Relationship between percentage muscle shortening and percentage lumen narrowing: a) in diameter; and b) in cross-sectional area of bronchial segments $(n=16)$. Narrowing in each segment was recorded to a dose of $\mathrm{ACh}\left(10^{-5}-10^{-2} \mathrm{M}\right)$ and then the segment was fixed for histological processing and estimation of muscle shortening. Predicted curves shown take the inner wall area into account (dashed lines, see Methods). ( $r=0.8162$ for diameter; $r=0.8445$ for area; $p<0.001$ ).

The correlation coefficients for the fitted curve were 0.8445 for muscle shortening against area, and 0.8162 for diameter $(\mathrm{n}=16 ; \mathrm{p}<0.001)$.

\section{Transmural pressure on narrowing}

Increasing transmural pressure from -8 to $20 \mathrm{cmH}_{2} \mathrm{O}$ caused an increase in the luminal diameter of relaxed bronchi of $42 \%$, i.e. from $2.35 \pm 0.09$ to $3.35 \pm 0.21 \mathrm{~mm}$ $(\mathrm{p}<0.001)$. The absolute narrowing response to EFS also increased with greater transmural pressures, with maximum narrowing present by $10 \mathrm{cmH}_{2} \mathrm{O}$ pressure (fig. 7) $(\mathrm{p}<0.05)$. However, expressed as a percentage of the relaxed diameter, narrowing did not change significantly with pressure. It was $23.2 \pm 3.4,26.7 \pm 5.3,30.7 \pm 7.5$ and $29.5 \pm 6.0 \%$ at $-8,0,10$ and $20 \mathrm{cmH}_{2} \mathrm{O}$, respectively, $(\mathrm{p}>0.05)$. 


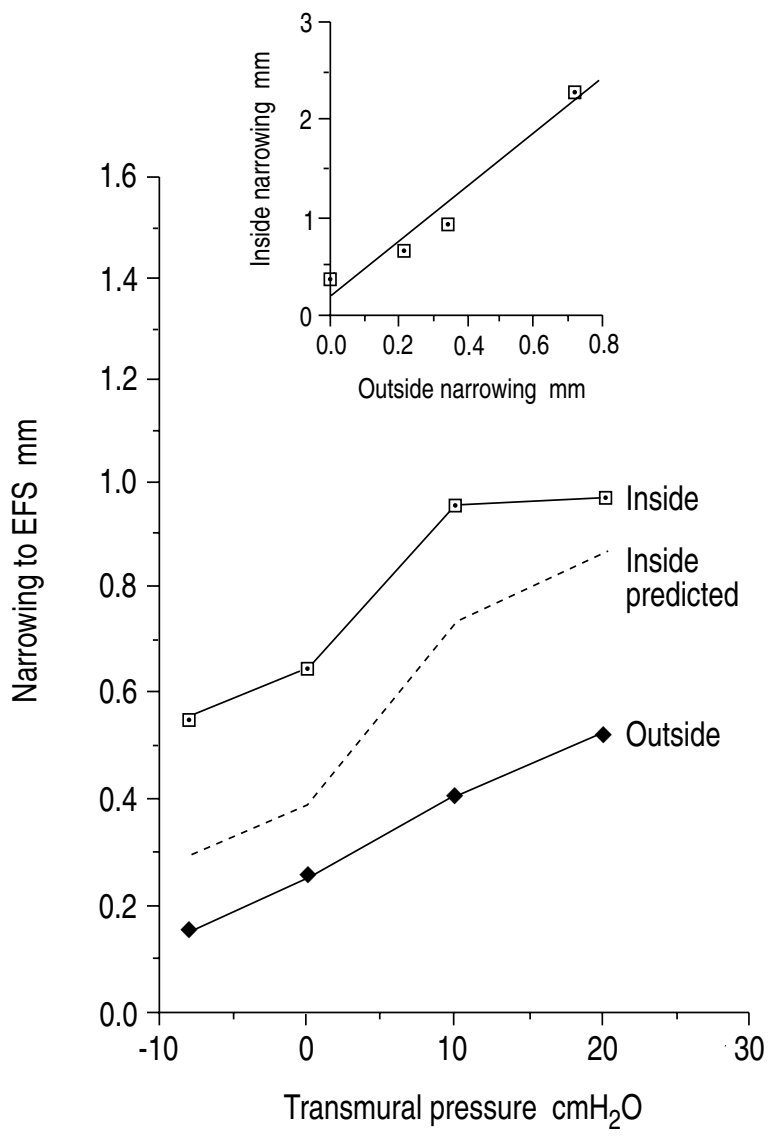

Fig. 7. - Narrowing of luminal (inside) and adventitial (outside) surfaces of bronchial segments to electrical field stimulation (EFS) at different transmural pressures. The adventitial diameter was measured through a calibrated dissecting microscope placed above the segment and the lumen diameter was recorded by endoscopic video-imaging. The predicted movement of the luminal surface (dashed line) takes the total wall area into account (see Methods). There were significant differences in narrowing responses at different pressures and between narrowing inside and out (analysis of variance (ANOVA) $\mathrm{p}<0.05-\mathrm{p}<0.001$; $\mathrm{n}=4)$. Inset: inside and outside narrowing to acetylcholine $\left(10^{-5}\right.$ $10^{-2} \mathrm{M}$ ) where each point is data from a single bronchial segment.

\section{Inside versus outside narrowing}

Concurrent recordings of the mucosal (inside) and adventitial (outside) margins of the bronchial wall indicated that the inside narrowed more than the outside to EFS and to ACh $(\mathrm{p}<0.001)$ (figs 7 and 8). For example, EFS reduced the inside diameter by $0.62 \pm 0.14 \mathrm{~mm}$, but the outside was reduced by only $0.14 \pm 0.05 \mathrm{~mm}$ at $0 \mathrm{cmH}_{2} \mathrm{O}$ transmural pressure. Figure 7 also indicates a predicted movement of the luminal wall to EFS, taking the total wall area of the bronchial segments into account (see Methods). The difference between the measured and predicted movement of the lumen was greatest at low transmural pressures, but it became less as the pressure approached $20 \mathrm{cmH}_{2} \mathrm{O}$.

\section{Discussion}

In this study, we directly visualized in vitro airway narrowing when flow through the lumen was partially and maximally reduced. Previously, responsiveness of individual bronchial segments was defined from the flow rate and flow reduction to provocation [14-17]. Ultrasound imaging techniques have failed to adequately visualize narrowing in vitro [19]. To obtain quantitative information about luminal dimensions in one particular plane, or region, it was necessary to apply a marker to the mucosal surface. The length of the segment was fixed, so that the distance between the dyed region of the mucosa and the endoscope lens did not change during muscle activation, ensuring that the change in the recorded image was due only to lumen narrowing. It was possible to define diameter as well as lumen cross-sectional area, because the lumens, of the bronchi, both unstimulated and contracted, were almost circular. The small spaces between the invaginations in the bronchial circumference caused by the mucosal ridges seen endoscopically were not included in the traced region used for analysis, but from histological sections these were minor in area. Furthermore, the areas of the lumen recorded endoscopically agreed closely with the luminal areas determined, subsequently, after histological preparation. When fluidfilled these interstices may not contribute to bulk flow through the airway lumen [13].

The present findings with direct visualization show that pig bronchus narrows by approximately $50 \%$ of diameter or $70 \%$ area to maximum cholinergic stimulation with $\mathrm{ACh}$, at $0 \mathrm{cmH}_{2} \mathrm{O}$ transmural pressure. In vivo, radiographs taken at functional residual capacity (FRC) suggest a maximum $20-30 \%$ narrowing in the pig, or $40-50 \%$ in the dog [1-3]. Altering the transmural pressure, in the present experiments, from -8 to $20 \mathrm{cmH}_{2} \mathrm{O}$ by applying pressure luminally stretched the circumference of the isolated bronchial segments by $42 \%$, and caused greater absolute narrowing responses to EFS; results best explained by movement of the smooth muscle along its length-tension curve. Maximum narrowing was evident by about $10 \mathrm{cmH}_{2} \mathrm{O}$ transmural pressure. However, the increase in narrowing with pressure was nearly matched by the increased resting circumference, so that the percentage narrowing was about $23 \%$ at $-8 \mathrm{cmH}_{2} \mathrm{O}$ and peaked at $30 \%$ at $10 \mathrm{cmH}_{2} \mathrm{O}$. A relationship between transpulmonary pressure and percentage narrowing of bronchi in vivo appears sharper than this [2]. It is highly unlikely that the narrowing of the lumen in vitro was substantially altered by removing parenchymal attachments and adventitial constraint, because the movement of the adventitial surface of the bronchial segments during stimulation was slight. We suggest that elastic forces acting on the outer surface of cartilaginous bronchi at normal lung volumes in vivo exert relatively little effect on responsiveness.

We measured narrowing in conjunction with responses defined by flow changes to stimulation. For the most part, the narrowing responses measured by endoscopy in this study agree closely with predictions assuming that flow is Poiseuillian. ACh $10^{-2} \mathrm{M}$ reduced the flow of Krebs solution through the lumen of perfused bronchi close to zero, and this was associated with a halving of the luminal diameter. To all-intents-and-purposes, this is airway closure and if present in "resistance" airways 
in vivo may lead to a dose-response curve similar to that seen in asthmatic or hyperresponsive individuals, i.e. where there is no flattening or plateau at the top end of the dose-response curve. This is consistent with recent findings in airways $3 \mathrm{~mm}$ or less [14] which, in addition, indicate that larger bronchi (e.g. $5 \mathrm{~mm})$ do demonstrate a plateau, presumably because muscle shortening is unable to cause sufficient occlusion of the lumen, even when it is maximally activated.

The present study establishes a relationship between bronchial diameter and smooth muscle shortening, that had not before been defined experimentally. One of the assumptions implicit in the calculation of muscle shortening, i.e. constancy of wall area during contraction [18], was validated in this study. The relationship between muscle shortening and airway narrowing shown here was independent of experimental conditions, e.g. transmural pressure, but may be dependent on wall thickness [9-11]. Indeed, the experimental data fit closely to a predicted relationship, which takes the wall thickness (measured from histological sections) into account. Factors that change the wall area or the forces generated by the smooth muscle will predictably alter the relationship defined here. Our data show that airway closure is produced by about $35 \%$ muscle shortening. Simulations by WIGGS and coworkers $[10,11]$ estimated that $30 \%$ muscle shortening causes about a sixfold increase in resistance in human 3-4 mm airways, and 40\% muscle shortening about a tenfold increase. Our experimentally derived data on similar sized bronchi show that resistance will be increased more than this. The relative wall area used in the simulation was slightly less (0.15) than that of the pig bronchi studied here. Models of airway narrowing may possibly underestimate the influence of the inner airway wall, and of mucosal folding, on flow.

The video recording system permitted the visualization of several structural properties of the airway lumen, as well as of dynamic responses associated with bronchoconstriction. The mucosal foldings seen via the endoscope in fresh bronchial segments are most probably the same as those seen in fixed tissue, since the numbers were the same (about 24). The numbers did not change during bronchoconstriction, which may be due to their association with fixed structures, for example submucosal vessels [20]. It was suggested that bronchial hyperresponsiveness could be associated with a decrease in the number of folds in airways [12]. There are no measurements of the mucosal foldings in asthmatic and normal airways to see whether they differ, but the present findings indicate that such changes would require a restructuring of the airway mucosa and of the anchoring points for the mucosal folds.

The rapidity of the observed recovery response of segments after EFS and the recovery of lumen circularity indicate that the bronchial wall is quite stable, even when unsupported by a transmural pressure. We demonstrated that the various components of the bronchial wall have some independence of movement, as evidenced by the greater narrowing of the inner parts comprising the mucosa and the muscle, compared to the outer cartilaginous parts. The difference between inside and outside narrow- ing cannot be accounted for through simple occupation of the lumen by the airway wall. VAN HAYEK [21] described submucosal separation in human bronchial sections, and independent movement of different cartilage pieces in response to deforming pressure has been noted in the cat bronchus [22]. The present findings were made by the simultaneous imaging on the inside and the outside of the bronchial segment. Bronchograms recorded in previous studies imaged only the lumen [1-3]. The phenomenon has not generally been recognized in airways studied histologically from lungs in vivo, possibly because the effect may be minimized when the lung is inflated to high pressure for fixation [10]. An effect of inflation pressure on the extent of wall separation in stimulated bronchi was described in the present study. In our experimental set-up, the spaces produced during narrowing may have been taken up with bathing solution, but if a similar situation occurs in vivo then the space may be partly taken by vascular dilatation or oedematous fluid. A phenomenon of wall uncoupling may be important in an action of inflammatory cell-derived proteinases and in excessive airway narrowing.

The application of video-imaging via an endoscope to bronchial narrowing has allowed us to visualize the bronchial lumen, and to directly determine cholinergic responsiveness in terms of lumen narrowing and the smooth muscle shortening that brings this about. Halving of the luminal diameter is produced when the smooth muscle shortens by about $35 \%$, and this causes perfusion through the lumen to stop. At low transmural pressure, muscle contraction also causes some separation of the inner parts of the bronchial wall from the cartilaginous adventitia.

\footnotetext{
Acknowledgements: The authors thank P. Grayson for technical assistance and G. Yates for mathematical help.
}

\section{References}

1. Murphy TM, Roy L, Phillips J, et al. Effect of maturation on topographic distribution of bronchoconstrictor reponses in large diameter airways of young swine. Am Rev Respir Dis 1991; 143: 126-131.

2. Shioya T, Munoz NM, Leff AR. Effect of resting smooth muscle length on contractile response in resistance airways. J Appl Physiol 1987; 62: 711-717.

3. Cabezas GA, Graf PD, Nadel JA. Sympathetic versus parasympathetic nervous regulation of airways in dogs. J Appl Physiol 1971; 31: 651-655.

4. Gunst SJ, Warner DO, Wilson TA, Hyatt RE. Parenchymal interdependence and airway response to methacholine in excised dog lobes. J Appl Physiol 1988; 65: 24902497.

5. Murtagh PS, Proctor DF, Permutt S, Kelly B, Evering S. Bronchial closure with mecholyl in excised dog lobes. J Appl Physiol 1971; 31: 409-415.

6. McNamara AE, Muller NA, Okazawa JA, Wiggs BR, Paré PD. Airway narrowing in excised canine lungs measured by high-resolution computed tomography. $J$ Appl Physiol 1992; 73: 307-316.

7. Brown RH, Herold CJ, Hirshman CA, Zerhouni EA, 
Mitzner W. In vivo measurements of airway reactivity using high-resolution computed tomography. Am Rev Respir Dis 1991; 144: 208-212.

8. Okazawa M, Paré P, Road J. Tracheal smooth muscle mechanics in vivo. J Appl Physiol 1990; 68: 209219.

9. James AL, Paré PD, Hogg JC. The mechanics of airway narrowing in asthma. Am Rev Respir Dis 1989; 139: 242-246.

10. Wiggs BR, Moreno JC, Hilliam C, Paré PD. A model of the mechanics of airway narrowing. J Appl Physiol 1990; 69: 849-860.

11. Wiggs BR, Bosken C, Paré PD, James A, Hogg JC. A model of airway narrowing in asthma and in chronic obstructive pulmonary disease. Am Rev Respir Dis 1992; 145: 1251-1258.

12. Lambert RK. Role of bronchial basement membrane in airway collapse. J Appl Physiol 1991; 71: 666673.

13. Yager D, Butler JP, Bastacky J, Israel E, Smith G, Drazen JM. Amplification of airway constriction due to liquid filling of airway interstices. J Appl Physiol 1989; 66: 2873-2884.

14. Mitchell HW, Sparrow MP. Increased responsiveness to cholinergic stimulation of small diameter cartilaginous bronchi compared to large. Eur Respir J 1994; 7: 298-305.
15. Mitchell HW, Willet KE, Sparrow MP. Perfused bronchial segment and bronchial strip: narrowing $v s$ isometric force by mediators. J Appl Physiol 1989; 66: 2704-2709.

16. Omari TI, Sparrow MP, Mitchell HW. Responsiveness of human isolated bronchial segments and its relationship to epithelial loss. Br J Clin Pharmacol 1993; 35: 357-365.

17. Hulsmann AR, Raatgeep HR, Bonta IL, Stijinen T, Kerrebijin KF, de Jongste JC. The perfused human bronchiolar tube characteristics of a new model. $J$ Pharmacol Meth 1992; 28: 29-34.

18. James AL, Hogg JC, Paré PD. The use of internal perimeter to compare airway size and to calculate smooth muscle shortening. Am Rev Respir Dis 1988; 138: 136-139.

19. Iizuki K, Dobashi K, Houjou S, Saki H, Itoh K, Nakazawa T. Evaluation of airway smooth muscle contractions in vitro by high-frequency ultrasonic imaging. Chest 1992; 102: 1251-1257.

20. Mitzner W, Wagner E. Bronchial vessels in the submucosa precipitate buckling of the airway epithelium. Am Rev Respir Dis 1993; 147: A507.

21. Van Hayek H. In: The Human Lung. New York, Hafner, 1960.

22. Olsen CR, Stevens AE, Pride NB, Staub NC. Structural basis for decreased compressibility of constricted tracheae and bronchi. J Appl Physiol 1967; 23: 35-39. 